\title{
Progesterone in Women with Bleeding in Early Pregnancy: A Bayesian re-analysis
}

\author{
James Uanhoro \\ uanhoro.1@osu.edu \\ Ohio State University
}

A randomized trial assessed the effect of progesterone in preventing pregnancy loss (Coomarasamy et al., 2019). The incidence of live births after at least 34 weeks of gestation (primary outcome) was $74.7 \%$ (1513 of 2025 women) in the progesterone group, and $72.5 \%$ (1459 of 2013 women) in the placebo group. Using standard frequentist methods, the authors concluded that the treatment effect was not statistically significant, $p=.081^{1}$

The motivation for this commentary is the Bayesian re-analysis of these data by Gronau and Wagenmakers (2019). I do not wish to comment on their commentary, but I find a different approach to re-analysis of these data to be of more value to the reader. Ryan, Harrison, Pearse, and Gates (2019) recently demonstrated the effect of using a Bayesian analysis to interpret the findings of clinical trials. I borrowed from their approach to reanalyze the primary outcome of the progesterone study; I was specifically interested in re-producing their Figure 3 for these data. In their Figure 3, they communicated the probability that the treatment effect exceeded different thresholds of effect size. For the re-analysis of the progesterone study, I assumed the data were binomial, and conducted a logistic regression. The prior for the treatment effect on the logit scale was normal with mean 0 and standard deviation $0.352^{2}$ The prior standard deviation for the intercept on the logit scale was normal with mean 0 and standard deviation $2.043^{3}$

Figure 1 shows the probability that progesterone had an average effect exceeding varying thresholds, as well as the posterior median for the observed effect for the different effect size measures. For example, the probability that progesterone was effective was about $95 \%$, i.e. $\operatorname{Pr}(\mathrm{OR} / \mathrm{RR}>1) \approx 95 \%$ or $\operatorname{Pr}(\mathrm{RD}>0) \approx 95 \%$. However, the probability that the odds ratio for progesterone exceeded 1.2 was under $20 \%$. Similarly, the probability that progesterone increased the probability of live births by more than $5 \%$ was under $20 \%$. Different readers can evaluate this drug based on the information in Figure 1 . If the reader is looking for any drug that is at least effective, then there is evidence in favour of using progesterone. However, if the reader needs a drug that will substantially improve the chance of live births, then the evidence is not in favour of progesterone. For a discussion of this approach to interpreting evidence, listen to Frank Harrell's appearance on the Plenary Sessions podcast. ${ }^{4}$

\footnotetext{
${ }^{1}$ My own analysis using a binomial logistic model, linear model or quasi-Poisson model resulted in $p=.11$.

${ }^{2}$ Precisely, the prior standard deviation was $\ln (2) / \Phi^{-1}(.975)$, where $\Phi^{-1}(\cdot)$ is the standard normal quantile function. I a-priori assumed a $95 \%$ chance of a treatment odds ratio between $1 / 2$ and 2 . In an earlier version of this paper, I mistakenly wrote that the prior standard deviation was $2 / \Phi^{-1}(.975)$.

${ }^{3}$ Precisely, the prior standard deviation was $4 / \Phi^{-1}(.975)$. This prior a-priori assumes a $95 \%$ chance that the incidence of live births without treatment was between $1.8 \%$ and $98.2 \%$.

${ }^{4}$ https://www.fharrell.com/talk/pspodcastbayes/
} 
Probability progesterone had an effect exceeding varying effect thresholds

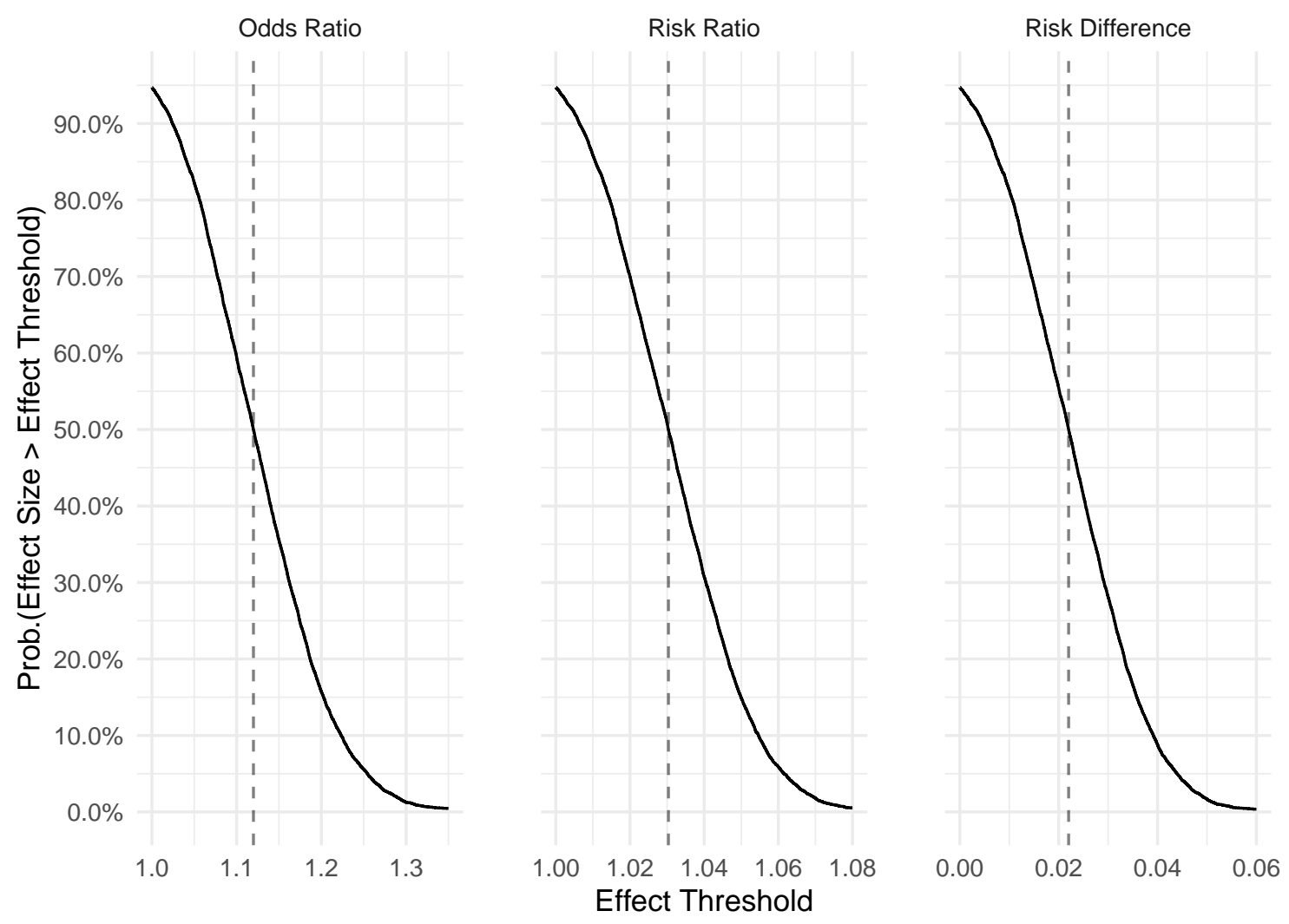

Figure 1. Probability that progesterone had an effect exceeding varying thresholds. The dashed vertical line was the median observed effect. To compute the probability on the y-axis, we computed the posterior samples for the three different effect sizes. We next counted the number of times the observed values in each posterior sample exceeded varying values of each effect size measure. The code to perform the analysis and produce the chart is available here: https://osf.io/sb2wx/.

A more complete analysis would include covariates and moderators, as the effect of progestorone likely varies by the person using the drug.

\section{References}

Coomarasamy, A., Devall, A. J., Cheed, V., Harb, H., Middleton, L. J., Gallos, I. D., .. Jurkovic, D. (2019, may). A Randomized Trial of Progesterone in Women with Bleeding in Early Pregnancy. New England Journal of Medicine, 380(19), 1815-1824. doi: 10.1056/NEJMoa1813730

Gronau, Q. F., \& Wagenmakers, E. (2019). Progesterone in Women with Bleeding in Early Pregnancy: Absence of Evidence, Not Evidence of Absence. doi: 10.31234/osf.io/etk7g

Ryan, E. G., Harrison, E. M., Pearse, R. M., \& Gates, S. (2019, mar). Perioperative haemodynamic therapy for major gastrointestinal surgery: The effect of a Bayesian approach to interpreting the findings of a randomised controlled trial. BMJ Open, 9(3), e024256. doi: 10.1136/ bmjopen-2018-024256 